\title{
THE INFLUENCE OF TRAINING ON ENDURANCE AND BLOOD LACTATE CONCENTRATION DURING SUBMAXIMAL EXERCISE
}

\author{
R. MAYES, BSc, MPhil, A. E. HARDMAN, MSc, PhD and C. WILLIAMS, BSc, MSc, PhD \\ Department of Physical Education and Sports Science, University of Technology, Loughborough, Leics. LE11 $3 T U$
}

\begin{abstract}
The purpose of this study was to examine the effect of short-term training on maximum oxygen uptake $\left(\mathrm{VO}_{2} \mathrm{max}\right)$ and two different measures of endurance performance. Endurance was determined for 15 female subjects (7 training, 8 control) as (1) exercise time to exhaustion at $80 \% \mathrm{VO}_{2} \max (\mathrm{T} 80 \%)$ and (2) the highest relative exercise intensity tolerable during a 30 minute test (T30 $\mathrm{min}$ ), before and after a 6-week training period. In addition, $\dot{\mathrm{V}}_{2}$ max and the work rate equivalent to a blood lactate concentration of $4 \mathrm{mmol} . \mathrm{I}^{-1}$ (OBLA) were determined. Maximum oxygen uptake increased by $24 \%$ ( $p<0.01$ ) for the training group (TG) and $7 \%(p<0.01)$ for the control group (CG). Cumulative average work rate (CAWR) during T30 min increased by $25 \%$ for the TG while there was no change for the CG. No significant difference was found pre- and post-training in the $\% \mathrm{VO}_{2}$ max (estimated from CAWR) at which the TG and CG performed T30 min. Exercise time to exhaustion on T80\% increased by $347 \%$ ( $p<0.01$ ) and $16 \%$ (NS) for the TG and the CG respectively. Good correlations were found between $\dot{\mathrm{VO}}_{2}$ max and CAWR (W) (pre-training $r=0.84$; post-training $r=0.83$ ), OBLA (W) and CAWR (W) (pre-training $r=0.89 ;$ posttraining $r=0.88$ ) and change in endurance time and the change in submaximal blood lactate concentration ( $r=0.70$, $p<0.01$ ). The results of this study suggest that the ability to sustain a high relative exercise intensity is not enhanced following short-term training.
\end{abstract}

Key words: Training, Endurance, Maximum oxygen uptake

\section{INTRODUCTION}

The possession of a high maximum oxygen uptake $\left(\mathrm{V}_{2}\right.$ max) is a prerequisite for successful endurance performance since the strong linear relationship between oxygen uptake and speed of running, swimming or cycling means that those individuals with a high $\mathrm{V}_{2}$ max should be able to maintain a higher absolute work rate than those with a low $\dot{\mathrm{V}}_{2}$ max. Increases in $\dot{\mathrm{VO}}_{2} \max$ as a result of endurance training, may thus be one reason for an increase in endurance with training, the change in $\mathrm{VO}_{2}$ max being dependent on factors such as intensity, frequency and duration of training, and the subject's initial fitness level.

Despite the fact that $\dot{\mathrm{V}}_{2}$ max may be increased by training, its adoption as an indicator of endurance fitness has been questioned. Firstly, it has been found to be largely predetermined genetically (Klissouras et al, 1973; Lortie et al, 1982) and secondly, training-induced changes in endurance performance may be independent of changes in $\dot{\mathrm{V}}_{2} \max$ (Williams and Nute, 1986).

An alternative description of an individual's training status may be obtained by assessing the individual's ability to sustain an exercise intensity that demands a high percentage of his or her $\mathrm{VO}_{2}$ max. This is because $\mathrm{VO}_{2}$ max describes the whole body's capacity to take up oxygen, while the fractional utilisation of $\dot{\mathrm{VO}}_{2} \max \left(\% \mathrm{VO}_{2} \max \right)$ over a given period of time reflects the metabolic characteristics of the working muscles and specifically their capacity for aerobic metabolism. This ability to sustain a high $\% \mathrm{VO}_{2}$ max has previously been examined in two ways, either by measuring the exercise time to exhaustion at a given $\% \dot{\mathrm{VO}}_{2} \max$ (Hardman, 1982) or by estimating the highest $\% \dot{\mathrm{VO}}_{2}$ max sustainable during a given period of time from measurements of the average work rate (Mayes, et al, 1985).

Address for correspondence:

Miss R. Mayes

Department of Physical Education and Sports Science

University of Technology

Loughborough

Leics. LE11 3TU
Direct measurement of the concentration of blood lactate also provides important information concerning the performance capacity of the individual. Researchers have found that the exercise intensity corresponding to a given blood lactate concentration, i.e. $4 \mathrm{mmol}^{-1} \mathrm{I}^{-1}$ or the onset of blood lactate accumulation (OBLA), not only appears to be a good predictor of endurance performance (Sjödin and Jacobs, 1981; Williams and Nute, 1983), but is also highly responsive to the influence of training (Sjödin et al, 1982).

Whilst it has been demonstrated repeatedly that both maximum oxygen uptake and the work rate corresponding to OBLA are higher during treadmill running than during cycling (Hermansen and Saltin, 1969; Jacobs and Sjödin, 1985), performance testing and training on a cycle ergometer has its advantages. This exercise mode allows exact measurement of the subject's work rate during performance tests and permits careful control of the intensity of the training sessions. The purpose of this study, therefore, was to examine the effect of short-term training on a cycle ergometer on both exercise time to exhaustion at a work rate equivalent to $80 \% \dot{\mathrm{VO}}_{2} \max (\mathrm{T} 80 \%)$ and the highest $\% \mathrm{VO}_{2}$ max tolerable during a 30 minute test (T30 min). A further objective was to assess the relationship between performance on these two tests and OBLA.

\section{EXPERIMENTAL METHODS}

Fifteen female subjects volunteered to take part in this study. Seven were assigned to the training group (TG), whilst eight acted as controls (CG). Two of the subjects within the TG were inactive prior to the study due to injury but the remaining subjects were all active. The ages and physical characteristics of the TG and CG are shown in Table I.

\section{TABLE I}

Physical characteristics of the subjects (mean \pm S.D.)

\begin{tabular}{lllll}
\hline & $\mathrm{n}$ & Height (cm) & Weight (kg) & Age (yrs) \\
\hline Training Group & 7 & $168.5 \pm 4.6$ & $64.4 \pm 8.3$ & $20.9 \pm 2.2$ \\
Control Group & 8 & $164.8 \pm 7.1$ & $62.5 \pm 8.8$ & $22.3 \pm 1.8$ \\
\hline
\end{tabular}




\section{Preliminary tests}

Each subject was familiarised fully with exercise on a Monark mechanically-braked cycle ergometer (model 864) before performing preliminary tests to determine (1) $\mathrm{VO}_{2}$ max and (2) the relationship between submaximal $\dot{\mathrm{VO}}_{2}$ and work rate and between blood lactate concentration and work rate.

Maximum oxygen uptake was determined by an incremental test where the work rate increased by $29 \mathrm{~W}$ every 3 minutes to exhaustion. The starting work rate ranged from $59 \mathrm{~W}$ to $176 \mathrm{~W}$ and was set for each individual so that exhaustion occurred within 10 minutes of exercise. Expired air samples were collected in Douglas bags during each submaximal stage and during the final minute of exercise for measurement of oxygen uptake and carbon dioxide production.

A submaximal incremental test consisting of 4 minutes of exercise at each of 4 increasing work rates (29W increments) was used to determine the relationships between work rate and oxygen uptake and work rate and blood lactate concentration. Work rates were set individually so that the initial work rate was approximately $50 \% \dot{\mathrm{VO}}_{2}$ max and final work rate in excess of $80 \% \dot{\mathrm{V}} \mathrm{O}_{2}$ max. Expired air samples were obtained during the last minute of each work rate. Thumb prick samples of capillary blood were obtained at rest and immediately after each expired air collection for the determination of blood lactate concentration. Each of the duplicate $25 \mu \mathrm{l}$ blood samples was deproteinised in $250 \mu$ l of $2.5 \%$ perchloric acid, centrifuged, frozen, and stored at $-20^{\circ} \mathrm{C}$. Analysis for blood lactate concentration was performed using the methodology described by Maughan (1982). A curve relating blood lactate concentration to the work rate was produced for each individual from which the work rate corresponding to a blood lactate concentration of $4 \mathrm{mmol} . \mathrm{I}^{-1}$ (OBLA) was interpolated.

\section{Endurance tests}

The two endurance tests, designated T80\% and T30 min, were both peformed on the same cycle ergometer. Pedal frequency was monitored continuously throughout the tests by a precision generator (R.S. Components Ltd.) attached to the flywheel of the cycle ergometer. Voltage output proportional to the flywheel speed was fed into a BBC microcomputer, via an analogue-to-digital converter, and used in conjunction with frictional force to calculate work rate.

For both the endurance tests subjects reported to the laboratory after an overnight fast. Each test was preceded by the collection of a resting blood sample and a 4-minute warm-up on the cycle ergometer at a work rate equivalent to $50 \% \mathrm{~V}_{2} \max$.

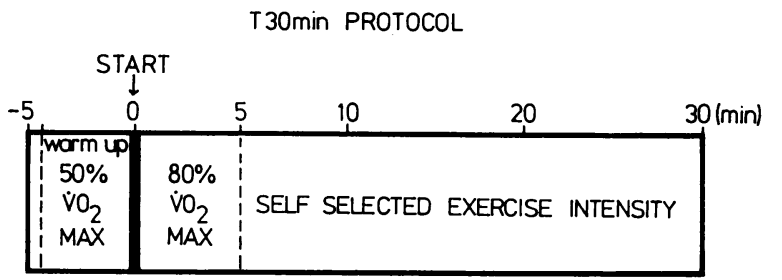

$\begin{array}{lllll}\text { EXP. AIR } & \uparrow & \uparrow & \uparrow & \uparrow \\ \text { THUMB } & \uparrow & \uparrow & \uparrow & \uparrow\end{array}$

Fig. 1: A schematic representation of the 30 minute test protocol (T30 min).

\section{0-Minute Test (T30 $\mathrm{min}$ )}

For the first 5 minutes of the test the subjects were required to exercise at a work rate equivalent to $80 \%$ of their $\mathrm{VO}_{2}$ max, adopting a pedal frequency of $60 \mathrm{rpm}$. For the remaining 25 minutes they were free to exercise at a work rate of their choice (by altering pedal frequency), with the aim of performing as much work as possible in the time available.

At the end of the test the computer calculated the average work done per minute of the exercise. This was referred to as the cumulative average work rate (CAWR). Using individual regression equations describing the relationship between $\dot{\mathrm{VO}}_{2}$ and work rate, the estimated oxygen cost for T30 min was calculated from the CAWR value. When expressed as a percentage of the subject's $\mathrm{VO}_{2}$ max, it provided a measure of the average $\% \dot{\mathrm{VO}}_{2}$ max that the subject had tolerated during T30 $\mathrm{min}$.

Four expired air collections and four samples of capillary blood were also taken during the test for the measurement of oxygen uptake and carbon dioxide production and the determination of blood lactate concentration. A schematic representation of the test protocol can be seen in Fig. 1.
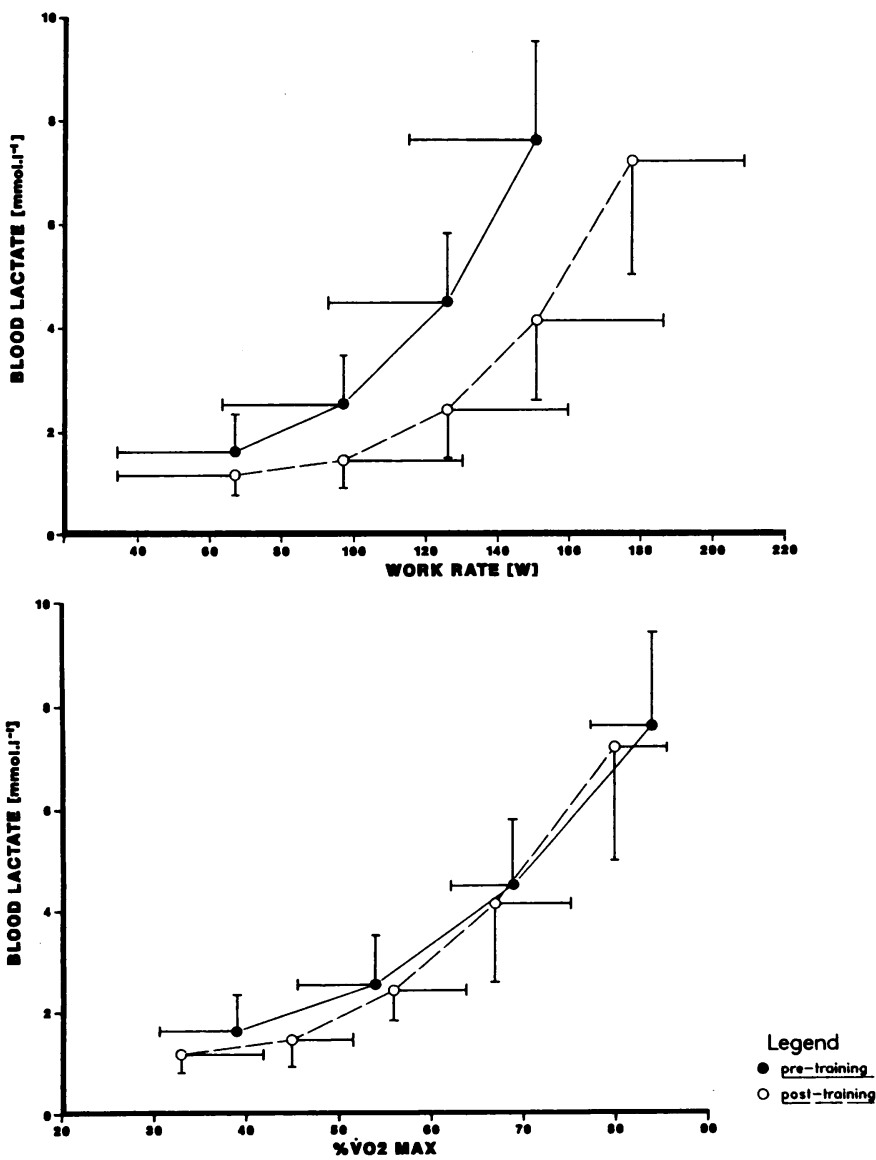

Fig. 2: The relationship between submaximal blood lactate concentration (mmol.1-1) and work rate $\mathrm{W}$ and $\% \mathrm{VO}_{2}$ max) for the training group pre-and post-training (mean \pm S.D.).

\section{$80 \% \mathrm{VO}_{2} \max$ Test (T80\%)}

Following the standardised warm-up the subjects were required to exercise to exhaustion at a work rate equivalent to $80 \%$ of their $\dot{\mathrm{VO}}_{2}$ max, adopting a pedal frequency of $60 \mathrm{rpm}$. The test was stopped when the subject could no longer maintain the required work rate. Expired air samples were taken at 5 minutes and at regular intervals during the test to confirm the relative exercise intensity. Thumb prick samples of capillary blood were obtained at rest, 
immediately following the expired air collection at 5 minutes and after the final minute. of exercise for the determination of blood lactate concentration.

\section{Training}

Each subject trained on the cycle ergometer 3 times a week, for 6 weeks. The training intensity was initially that used for $\mathrm{T} 80 \%$ i.e. $80 \% \mathrm{VO}_{2}$ max and the subjects were required to train for a maximum of $\mathbf{3 0}$ minutes per session. When the subjects had completed 30 minutes at this work rate on three occasions, the work rate was increased by either $15 \mathrm{~W}$ or $29 \mathrm{~W}$ according to body weight. During the training period both the TG and the CG maintained their normal physical activity patterns. After the 6-week training period both groups repeated the pre-training tests at the same work rates.

\section{Statistical Analysis}

Comparisons between the TG and the CG were evaluated using Student's t-test for independent means. Within-group comparisons pre- and post-training were made using Student's t-test for correlated means. Relationships between variables was assessed using the Pearson Product Moment correlation coefficient.

\section{RESULTS}

\section{Preliminary tests}

Table II shows a summary of the physiological characteristics of the TG and the CG pre- and post-training. No significant differences were found between the two groups on any of the variables measured in the pre-training tests.

In the post-training tests both the TG and the CG increased their $\dot{\mathrm{VO}}_{2}$ max values (I. $\left.\mathrm{min}^{-1}\right)$ significantly $(24 \%$ and $7 \%$ respectively) but the increase for the TG was significantly greater than that of the CG in both absolute ( $p$ $<0.05)$ and relative $(p<0.01)$ terms. Although blood lactate concentration for the TG was decreased significantly in the post-training incremental test at work rates 2,3 , and 4, there was no change in the blood lactate concentration when work rate was expressed relative to $\dot{\mathrm{VO}}_{2}$ max) $\left(\% \dot{\mathrm{VO}}_{2}\right)$ (Fig. $3)$. The results for the CG revealed no change in the blood lactate concentration at the same absolute work rates posttraining but there was a decreased concentration at the same relative work rate.

The absolute work rate at which OBLA occurred increased significantly for both the TG $(p<0.01)$ and the CG $(p<0.05)$ in the post-training tests. However, the TG

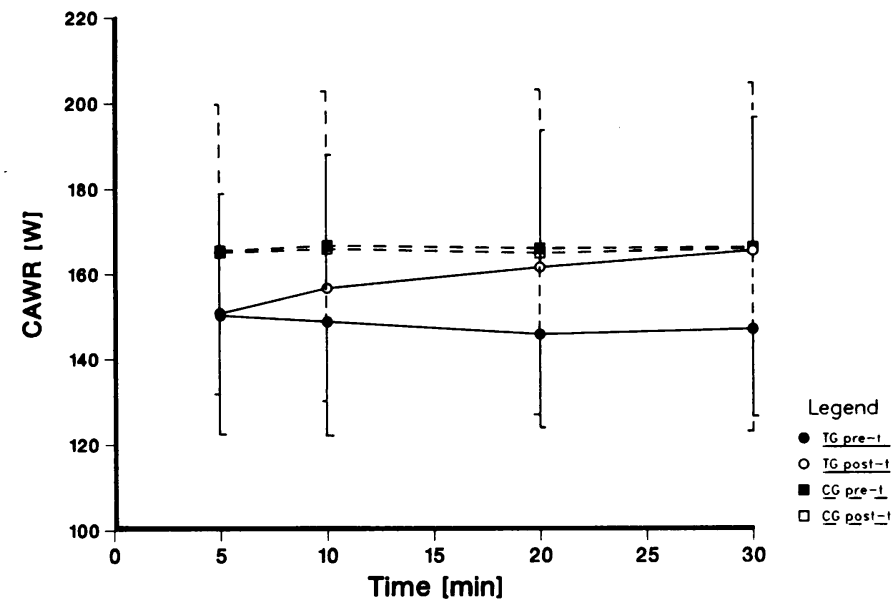

Fig. 3: Changes in mean ( + S.D.) Cumulative Average Work Rate (CAWR) for the training group (TG) and control group (CG) during T30 min pre-training (solid symbols) and post-training (open symbols).

increase in OBLA of $26 \%$ was significantly greater $(p<0.01)$ than that of the CG $(5 \%)$. When OBLA was expressed relative to the post-training $\mathrm{VO}_{2}$ max there was no change in the $\% \dot{V O}_{2}$ max at which OBLA occurred (OBLA \%) for either the TG or the CG. Post-training, OBLA \% was significantly higher for the CG than the TG $(p<0.05)$.

\section{Endurance Tests}

Results of T30 min (Table II) revealed a significant increase in CAWR for the TG during the post-training test $(p<0.01)$ while there was no change in CAWR for the CG (Fig. 3). No significant difference was found pre- and post-training in the $\% \dot{V O}_{2}$ max at which the TG and the CG performed T30 min, i.e. neither the TG nor the CG could exercise at a higher percentage of their $\dot{\mathrm{VO}}_{2}$ max during $\mathrm{T} 30 \mathrm{~min}$ than pre-training.

A summary of the blood lactate concentrations during the pre- and post-training endurance tests can be seen in Table III. Results from T30 min revealed that, post-training, blood lactate concentration was significantly lower for the TG following the initial 5 minutes standardised exercise period (CG, NS) whilst there was no significant difference pre- and post-training in the blood lactate concentrations after $\mathbf{3 0}$ minutes for either the TG or the CG (Fig. 4).

Exercise time to exhaustion on $\mathrm{T} 80 \%$ increased significantly by $347 \%$ for the TG post-training ( $p<0.01$ ) but there was no significant difference in the endurance time for the CG. Post-training, blood lactate concentrations were significantly lower throughout $\mathrm{T} 80 \%$ for both the TG ( $p<0.01)$ and the CG ( $<<0.05)$ (Table III).

TABLE II

A summary of the physiological characteristics of the training group (TG) and the control group (CG) pre- and post-training (mean \pm S.D.)

\begin{tabular}{|c|c|c|c|c|c|c|c|}
\hline & & \multirow{2}{*}{$\begin{array}{l}\mathrm{VO}_{2} \max \\
\text { I.min-1 }\end{array}$} & \multicolumn{2}{|c|}{ OBLA } & \multicolumn{2}{|c|}{$\mathrm{T} 30 \mathrm{~min}$} & \multirow{2}{*}{$\begin{array}{c}\text { T80\% Time } \\
\text { min }\end{array}$} \\
\hline & & & $\mathbf{w}$ & $\% \dot{V O}_{2} \max$ & CAWR W & $\% \dot{\mathrm{V}} \mathrm{O}_{2}$ maxt & \\
\hline \multirow[t]{3}{*}{ TG } & Pre & $2.42 \pm 0.50$ & $118 \pm 8$ & $66 \pm 8$ & $147 \pm 21$ & $80 \pm 7$ & $25.4 \pm 11.2$ \\
\hline & Post & $2.93 \pm 0.40^{b}$ & $146 \pm 26^{b}$ & $66 \pm 6$ & $165 \pm 31^{b}$ & $72 \pm 5$ & $97.7 \pm 35.9^{b}$ \\
\hline & $\%$ & 23.5 & 25.7 & -0.3 & 11.8 & -8.7 & 346.9 \\
\hline \multirow[t]{3}{*}{ CG } & Pre & $2.66 \pm 0.45$ & $141 \pm 32$ & $67 \pm 7$ & $166 \pm 34$ & $80 \pm 5$ & $30.6 \pm 12.4$ \\
\hline & Post & $2.87 \pm 0.56^{b}$ & $151 \pm 31^{\circ}$ & $73 \pm 6^{b}$ & $166 \pm 39^{b}$ & $77 \pm 7$ & $34.8 \pm 17.3$ \\
\hline & $\%$ & $7.0^{*}$ & $5.4^{* *}$ & $12.7^{*}$ & $-0.4^{* *}$ & -3.6 & $16.0^{* *}$ \\
\hline
\end{tabular}

Significantly different from pre-training, $a-p<0.05, b-p<0.01$

Significantly different from the TG, ${ }^{*} p<0.05,{ }^{*} p<0.01$

CAWR Cumulative Average Work Rate: $\uparrow$ Estimated from CAWR 


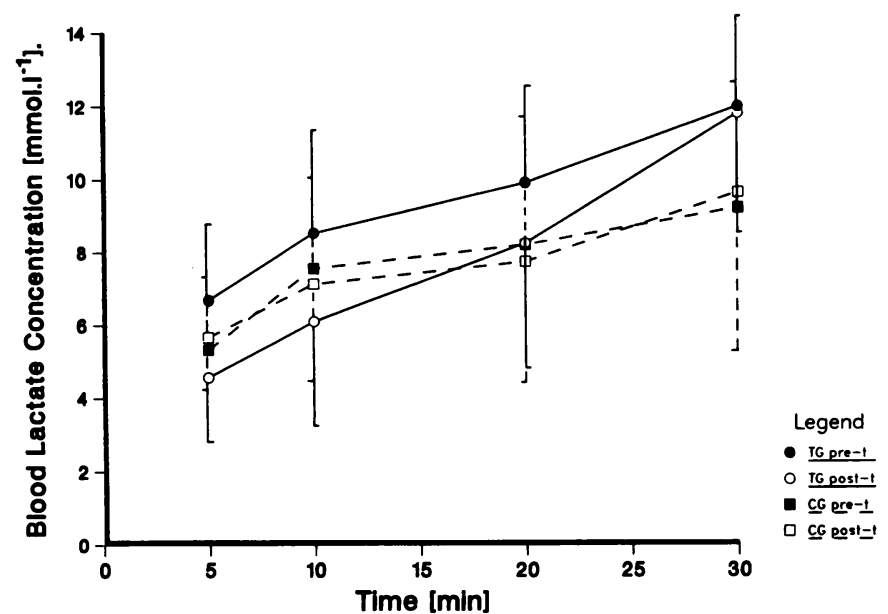

Fig. 4: Changes in mean ( \pm S.D.) blood lactate concentration (mmol.1-1) for the training group (TG) and control group (CG) during T30 min pre-training (solid symbols) and post-training (open symbols).

A summary of the relationships between selected physiological and performance variables can be seen in Table IV. There were strong correlations for the group as a whole between $\dot{\mathrm{V}}_{2} \max$ and OBLA (W) (pre-training $r=0.79$; post-training $r=0.77) ; V_{2} \max$ and CAWR (W) (pre-training $r=0.84$; post-training $r=0.83$ ); and OBLA $(W)$ and CAWR $(W)$ (pre-training $r=0.89$; post-training $r=0.88$ ), revealing that in this group of subjects OBLA was a slightly better predictor of CAWR than was $\mathrm{VO}_{2}$ max. Poor correlations were found between $\dot{\mathrm{VO}}_{2} \max$ and $\% \dot{\mathrm{VO}}_{2}$ max estimated from CAWR (pre-training $r=-0.21$; post-training $r=0.12$ ) and $\dot{\mathrm{VO}}_{2} \max$ and $\mathrm{T} 80 \%$ time (pre-training $r=0.08$; post-training $r=0.15$ ).

\section{TABLE III}

Blood lactate concentrations ( $\mathrm{mmol} . \mathrm{I}^{-1}$ ) during the two endurance tests preand post-training (mean \pm S.D.)

\begin{tabular}{lcccc}
\hline & \multicolumn{2}{c}{ TRAINING GROUP $(n=7)$} & \multicolumn{2}{c}{ CONTROL GROUP $(n=8)$} \\
\hline T30 min & $5 \mathrm{~min}$ & $30 \mathrm{~min}$ & $5 \mathrm{~min}$ & $30 \mathrm{~min}$ \\
pre & $6.68 \pm 2.09$ & $11.98 \pm 2.38$ & $5.22 \pm 1.05$ & $9.21 \pm 3.97$ \\
post & $4.57 \pm 1.88^{*}$ & $11.79 \pm 3.36$ & $5.56 \pm 1.65$ & $9.63 \pm 3.00$ \\
T80\% & $5 \mathrm{~min}$ & final min & $5 \mathrm{~min}$ & final min \\
pre & $6.55 \pm 0.98$ & $11.20 \pm 1.99$ & $6.16 \pm 1.37$ & $10.12 \pm 2.85$ \\
post & $4.26 \pm 1.32^{* *}$ & $7.32 \pm 3.03^{* *}$ & $5.36 \pm 1.07^{*}$ & $7.83 \pm 2.56^{*}$
\end{tabular}

Significantly different from pre-training, ${ }^{* *} p<0.01,{ }^{* *} p<0.05$

Although strong correlations were found between. $\mathrm{VO}_{2}$ max and OBLA (W) for the group as a whole both pre- and post-training, only a poor correlation was found between the percentage change in these two variables $(r=0.41, N S)$. The percentage change in $\dot{\mathrm{VO}}_{2}$ max for the TG, however, showed a strong inverse relationship with the pre-training $\dot{\mathrm{V}}_{2}$ max values $(r=-0.87, p<0.05)$. A strong inverse relationship was also found between percentage change in $\dot{\mathrm{VO}}_{2}$ max and the change in the $\% \dot{\mathrm{VO}}_{2}$ max sustained by the TG during T30 $\min (r=-0.88, p<0.01)$.

The percentage change in T80\% time was significantly related to both the change in OBLA (W) $(r=0.57, p<0.05)$ and the change in blood lactate at the fourth work rate in the submaximal incremental test $(r=0.70 ; p<0.01)$. In addition, a strong inverse relationship $(r=-0.88, p<0.01)$ was found between the relative exercise intensity of the post training T80\% (expressed as a percentage of the posttraining $\left.\dot{\mathrm{V}}_{2} \max \right)$ and the time to exhaustion $(n=15)$.
TABLE IV

Pearson Product Moment correlation coefficients for selected physical and physiological variables pre- and post-training $(n=15)$

\begin{tabular}{llcccc}
\hline & & & \multicolumn{2}{c}{ T30 min } & \multicolumn{2}{c}{ T80\% } \\
& & OBLA (W) & CAWR (W) & $\% \dot{V}_{2}$ maxt & Time (min) \\
\hline VO $_{2}$ max & pre & $0.79^{* *}$ & $0.84^{* *}$ & -0.21 & 0.08 \\
$($ I.min-1) & post & $0.77^{* *}$ & $0.83^{* *}$ & 0.12 & 0.15 \\
OBLA & pre & - & $0.89^{* *}$ & - & - \\
(W) & post & - & $0.88^{* *}$ & - & - \\
T80\% & pre & - & - & - & -0.15 \\
$\% \mathrm{VO}_{2}$ max & post & - & - & - & $-0.88^{* *}$ \\
\hline
\end{tabular}

** $p<0.01$

t estimated from CAWR

\section{DISCUSSION}

The results of this study revealed that, after six weeks of training, $\mathrm{VO}_{2}$ max increased significantly by $25 \%$ for the TG, with a range of $6-53 \%$. The magnitude in change in $\mathrm{VO}_{2} \max$ was influenced strongly by the pre-training value, with a strong correlation of $r=-0.87$ ( $p<0.05$ ) between percentage change and the initial $\dot{\mathrm{VO}}_{2} \max$ value. This finding is in keeping with reports in the literature which have found an inverse relationship between the initial value of $\dot{\mathrm{VO}}_{2}$ max and its percentage change after training (Pollock, 1973). The significant change in the $\mathrm{VO}_{2}$ max of the CG may be attributed to an increase in their normal physical activity patterns. Six of the control subjects were physical education students who, on returning to University after a 5-week vacation, were engaged in a physically demanding course during the training study period.

The training-induced change in $\mathrm{VO}_{2}$ max for the TG was paralleled by a similar change in the absolute work rate at which OBLA occurred ( $26 \pm 19 \%)$. There was, however, only a modest correlation between change in $\mathrm{VO}_{2}$ max and change in OBLA ( $r=0.41, N S)$ suggesting that these changes were independent of each other. As might be expected from their increase in $\mathrm{VO}_{2}$ max, the $\mathrm{CG}$ also showed a significant increase in the work rate at which OBLA occurred ( $p<0.05$ ) but the magnitude of change was significantly smaller than that of the TG.

Despite an increase in the absolute work rate corresponding to OBLA for the TG, there was no change in the $\% \mathrm{VO}_{2} \max$ at which it occurred. The improved $\mathrm{VO}_{2} \max$ may have accounted for the unaltered relationship between relative exercise intensity and blood lactate concentration despite the decrease seen in blood lactate concentration at a given absolute work rate. It is possible also that such results are specific to short-term training periods. Therefore, for relatively untrained subjects such as those involved in this study, an increase in the $\% \dot{\mathrm{VO}}_{2} \max$ at which OBLA occurs may only be apparent after long-term training.

The altered responses of the TG to the preliminary submaximal incremental test were also reflected in their performances in the post-training T30 $\mathrm{min}$. The absolute work rate at which the TG performed T30 min was significantly higher post-training despite the fact that they were unable to exercise at a higher $\% \dot{V}_{2}$ max. One of the factors influencing the $\% \dot{\mathrm{VO}}_{2}$ max that the subject could tolerate may have been the magnitude of the traininginduced change in $\dot{\mathrm{V}}_{2}$ max. For, when the percentage change in $\dot{\mathrm{VO}}_{2}$ max was correlated with the percentage change in the $\% \dot{\mathrm{VO}}_{2}$ max tolerated during $\mathrm{T} 30 \mathrm{~min}$ an 
inverse relationship was found between the two variables $\langle r=-0.88, p<0.01)$. This would imply that those individuals with a small change in $\mathrm{VO}_{2}$ max could tolerate a higher percentage of their new $\mathrm{VO}_{2}$ max than those with a large change, and suggests that the increase in the ability to sustain a high $\% \dot{V}_{2}$ max following training is influenced strongly by the extent to which $\mathrm{VO}_{2}$ max has altered.

Analysis of the blood lactate concentrations during T30 min revealed a significant increase in blood lactate concentration throughout the 30 minutes for the TG ( $p<0.01)$ and for the CG ( $p<0.05)$ both pre- and posttraining (Fig. 4). This increase occurred despite the fact that there was no significant increase in the work rate over the final $\mathbf{2 5}$ minutes in this test for either group pre-training, or the CG post-training (Fig. 3). This increase in lactate concentration, in the absence of an increase in work rate, may partly be accounted for by the high blood lactate concentrations already present after 5 minutes of exercise

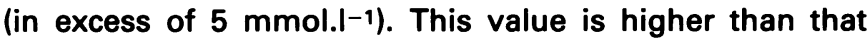
reported by Kindermann et al (1979) as the level at which blood lactate concentration begins to increase during exercise at a constant work rate. At this exercise intensity and above, an imbalance between lactate production and lactate clearance results in lactate accumulation. This would then prevent the subjects increasing their work rate during the remaining 25 minutes of the test and in some instances cause them to reduce it. A slightly lower blood lactate concentration at 5 minutes $\left(4.57 \mathrm{mmol} . \mathrm{I}^{-1}\right)$ was found for the TG in the post-training test. This value is closer to the $4 \mathrm{mmol}^{-\mathrm{I}^{-1}}$ value at which a steady state can be maintained for as long as 45-60 minutes without a further increase in lactate concentration (Kindermann et al, 1979).

The widely reported decrease in blood lactate concentration at a given absolute work rate as a result of training (Williams et al, 1967; Ekblom, 1969) was clearly evident for the TG after the first 5 minutes standardised exercise in the post-training T30 min. During the remaining 25 minutes of the test, however, it increased to a value similar to that recorded at the end of the pre-training test. So, despite a higher work rate post-training than pretraining, blood lactate concentration was similar at the end of both tests. In addition, since neither the $\% \mathrm{VO}_{2} \max$ at which the subjects performed T30 min nor the blood lactate concentrations at the end of this test differed significantly pre- and post-training the results of T30 min are consistent with those of the submaximal incremental test which showed no change in the $\% \dot{\mathrm{VO}}_{2}$ max at which blood lactate accumulation occurred. As a result, the ability to exercise at a higher $\% \dot{\mathrm{V}}_{2}$ max was not enhanced by training.

Although a strong correlation was found both pre- and post-training between $\dot{\mathrm{V}}_{2}$ max and CAWR for the group as a whole ( $r=0.84$ and $r=0.83$ respectively) there was an even stronger relationship between OBLA (W) and CAWR (pre-training $r=0.89$; post-training $r=0.88$ ). These correlations are similar to those reported by Sjödin and Jacobs (1981, $r=0.96)$; and Williams and Nute (1983, $r=-$ 0.88 ) for the relationship between OBLA and marathon and half-marathon performance times respectively. It would appear, therefore, that for this group of subjects OBLA (W) was a more sensitive predictor of performance on the 30 minute test than was $\dot{\mathrm{VO}}_{2}$ max.

One of the most dramatic changes in submaximal performance as a result of 6 weeks' training was the $347 \%$ increase in exercise time to exhaustion during $\mathrm{T} 80 \%$ demonstrated by the TG (CG, NS). Since T80\% was performed at the same absolute work rates pre- and posttraining, annd because all the subjects showed an increase in $\dot{\mathrm{VO}}_{2}$ max, the $\% \dot{\mathrm{VO}}_{2}$ max at which the subjects were exercising was an important determinant of endurance time. Although the pre-training work rate elicited $84 \%$ and $82 \% \mathrm{VO}_{2}$ max for the TG and CG respectively, post-training this same work rate represented only $68 \%$ of the TG posttraining $\mathrm{VO}_{2} \max$ and $79 \%$ of the $\mathrm{CG} \mathrm{VO}_{2}$ max. The strong inverse relationship between $\% \mathrm{VO}_{2}$ max and endurance time in the post-training test $(r=-0.88, p<0.01)$ indicated that those individuals whose work rate represented a low $\% \dot{V O}_{2}$ max were able to exercise for longer than those whose work rate represented a higher $\% \mathrm{VO}_{2}$ max.

The training-induced change in submaximal blood lactate concentration also appeared to be an important determinant of post-training $\mathrm{T} 80 \%$ time. When percentage change in endurance time and the decrease in blood lactate concentration at the fourth work rate in the submaximal incremental test were correlated a strong relationship, similar to that reported by Williams and Nute (1986) for running at $90 \% \dot{V O}_{2}$ max, was found $(r=0.70, p<0.01$ ). This implies that those individuals who showed the greatest metabolic adaptations within the muscles, as indicated by a decrease in blood lactate concentration, were able to increase their endurance time the most, and supports the suggestions that muscle metabolism dictates endurance (Davies, et al, 1981, 1982). Although there was also a modest correlation between change in OBLA (W) and percentage change in endurance time $(r=0.57 ; p<0.05$; $n=5$ ) it would appear that the changes in blood lactate concentrations were greatest at the exercise intensity at which the subjects had been training, i.e. the highest work rate in the submaximal test $\left(80 \% \mathrm{VO}_{2}\right.$ max $)$.

In conclusion, the major finding of this study was that after 6 weeks of training, the ability to exercise at a given absolute work rate was enhanced due to an increase in $\mathrm{VO}_{2}$ max and a reduced blood lactate concentration during submaximal exercise. However, the capacity to exercise at a high relative exercise intensity remained unchanged. Improvements in performance attributable to an increased ability to tolerate a high fraction of $\dot{\mathrm{V}}_{2}$ max may thus be a later event in the response to training.

\section{References}

Davies, K. J. A., Packer, L. and Brooks, G. A., 1981 "Biochemical adaptations of mitochondria, muscle, and whole-animal respiration to endurance training". Archives of Biochemistry and Biophysics 209: 539-554.

Davies, K. J. A., Packer, L. and Brooks, G. A., 1982 "Exercise bioenergetics following sprint training". Archives of Biochemistry and Biophysics 215: 260-265.

Ekblom, B., 1969 "Effect of physical training on oxygen transport system in man". Acta Physiol.Scand.Suppl. 328.

Hardman, A. E., 1982 "The influence of training on metabolic responses to submaximal exercise". Br.J.Sports Med. 16: 107.

Hermansen, L. and Saltin, B., 1969 "Oxygen uptake during maximal treadmill and bicycle exercise". J.Appl.Physiol. 26: 31-37.

Jacobs, I. and Sjödin, B., 1985 "Relationship of ergometer-specific $\mathrm{VO}_{2}$ max and muscle enzymes to blood lactate during submaximal exercise". Br.J.Sports Med. 19: 77-80.

Kindermann, W., Simon, G. and Keul, J., 1979 "The significance of the aerobic-anaerobic transition for the determination of work load intensities during endurance training". Eur.J.Appl.Physiol. 42: 25-34.

Klissouras, V., Pirnay, F. and Petit, J.-M., 1973 “Adaptation to maximal effort: genetics and age". J.Appl.Physiol. 35: 288.

Lortie, G., Bouchard, C., Leblanc, C., Temblay, A., Simoneau, J.-A., Theriault, G. and Savoie, J.-P., 1982 "Familial similarity in aerobic power". Human Biology 54: 801-812.

Maughan, R. J., 1982 "A simple rapid method for the determination of glucose, lactate, pyruvate, alanine, 3-hyroxybutyrate and acetoacetate on a single $20 \mu$ l blood sample". Clin.Chim.Acta 122: 231-240. 
Mayes, R., Hazeldine, R. J. and Williams, C., 1985 "A test of endurance fitness". J.Sports Sci. 3: 215-216.

Pollock, M. L., 1973 "The quantification of endurance training programs". In Exercise and Sports Science Reviews. Vol. 1 (ed. J. H. Wilmore), pp. 155-188. New York: Academic Press.

Sjödin, B. and Jacobs, I., 1981 "Onset of blood lactate accumulation and marathon running performance". Int.J.Sports Med. 2: 23-26.

Sjödin, B., Jacobs,I. and Svedenhag, J., 1982 "Changes in Onset of Blood Lactate Accumulation (OBLA) and muscle enzymes after training at OBLA". Eur.J.Appl.Physiol. 49: 45-57.
Williams, C. and Nute, M. L. G., 1983 "Some physiological demands of a halfmarathon race on recreational runners". Br.J.Sports Med. 17: 152-161.

Williams, C. and Nute, M. L. G., 1986 "Training induced changes in endurance capacity of female games players". In "Sports Science". Proceedings of the VII Commonwealth International Conference on Sport, Physical Education, Dance, Recreation and Health. (Ed.) Watkins, J., Reilly, T. and Burtwitz, L. pp. 11-17. London: Spon.

Williams, G., Wyndham, C. H., Kok, R. and von Rahden, M. J. E., 1967 "The effect of training on maximum oxygen uptake and anerobic metabolism in man". Int.Z.Angew.Physiol. 24: 18-23.

\title{
Title: \\ Editor: $\quad$ R. M. Jay \\ Publisher: $\quad$ Year Book Medical Publishers, Chicago and London. UK agents - Wolfe Medical Price: $€ 38.00 \quad$ ISSN 0815148577
}

\section{BOOK REVIEW}

The 1986 Year Book of Podiatric Medicine and Surgery is the second volume in a series of annual literature reviews for the Podiatric profession. It is also a useful reference book for other health care professions who are concerned with the health and disease, function and malfunction of the foot and thus their effects on the rest of the body. The editors have selected what they believe to be the "superlative papers" relating to the feet in print to date. Over 500 different medical and allied health journals have been surveyed by the publisher from November 1984 through to October 1985. From this vast collection of literature, the editors have chosen selected articles reviewing foot-related problems in the following areas:- Paediatrics, Biomechanics, Forefoot and Rearfoot problems, Ankle, Sports Medicine, Traumatology, Neurology, Radiology, Tumours, Infection, Dermatology, Systemic Diseases, Arthritis, Diabetes Mellitus, and Peripheral Vascular Disease. Many of the articles have associated Editorial comment, clarifying or explaining the importance/relevance of the work reviewed.

Each chapter is suffixed with a list of the articles abstracted. Unfortunately, this is not an alphabetical or author listing. However, a brief overview will enable the reader to locate an article of particular interest. The subject index and author index are to be found at the back of the book. Both are comprehensive and include some cross-references. For those of us concerned with aspects of foot-related medical care this reference book is essential. It provides a wealth of updating information at a glance, which the practitioner would normally find difficult to access. I can thoroughly recommend the 1986 Year Book of Podiatric Medicine and Surgery for your practice library.

Susanna K. Nickson

\section{BOOK REVIEW}

Title:

\section{THERAPEUTIC MODALITIES IN SPORTS MEDICINE}

Author:

\author{
W. E. Prentice
}

Publisher: $\quad$ Year Book Medical Publishers, Chicago and London. UK agents - Wolfe Medical 1985

Price: $£ 28.95 \quad$ ISBN 0801640253

This American text book comes as the answer to at least one doctor's prayer. As the preface somewhat immodestly remarks "the availability of this text fills a void that has existed for quite some time." No doctor engaged in sports medicine can avoid the, sometimes unpalatable, fact for long that physiotherapy is really quite important, not least in the minds of the athletes. The uncomfortable realisation is that no-one ever taught us about physio and, until now, I have never been able to find a readable book that introduces the mysteries.

Not only is this book readable, but it is obviously written by teachers. Each chapter sets out its aims and ends with a summary of the important points in the text. It starts with a discussion on pain and its perception and goes on to explanations of the basic principles of the various energy sources, before a detailed discussion of the various applications of infra red, ultrasound, short wave and ultra violet. Whilst manual traction is a whole art in itself, the chapter in this book gives a useful idea of the principles involved together with some sound practical advice. Massage has always seemed to me to be a sadly neglected art and $I$ am pleased to see that it too is recognised as a useful and reputable technique, against a background of the principles involved.

It must be fairly obvious that I liked the book. I like its content and I like its style. Essential reading for Doctors and a good introduction for student physios. Whilst it probably has little to teach the experienced sports physiotherapist, it is still a good read, even for them. 